\title{
An Experimental Study of Three-Dimensional Passive Dynamic Walking with Flat Feet and Ankle Springs
}

\author{
Terumasa Narukawa, Kazuto Yokoyama, \\ Masaki Takahashi and Kazuo Yoshida \\ Keio University \\ Japan
}

\section{Introduction}

Passive dynamic bipeds were first studied by McGeer (McGeer, 1990) as inspired by a bipedal toy described in (McMahon, 1984). Passive dynamic walkers can walk down a shallow slope without actuators and controllers(McGeer, 1990; 1993). McGeer has built passive walkers that exhibit steady motion using a Poincaré map, which he called as a stride function, to analyze the gaits(McGeer, 1990; 1993). This method is quite useful and is independent of the biped model. The key idea that he examined is the stability of the entire step-to-step motion, and not the local stability at every instance. This analysis is also rather useful for actuated bipeds (Hobbelen and Wisse, 2007).

Firstly, McGeer studied two elementary passive walking models derived from a wagon wheel. One model was a rimless wheel model on a slope, and the other a synthetic wheel model on level ground as shown in Fig. 1. The motion of the models is constrained to the sagittal plane. Each model captures the fundamental mechanism of passive dynamic walking.

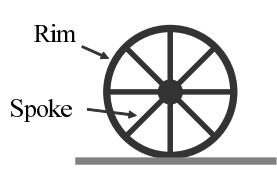

Wheel

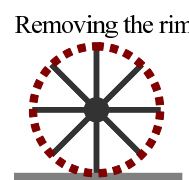

Removing all but two of the spokes

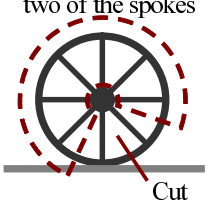

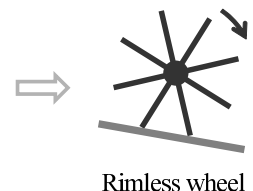

Rimless wheel

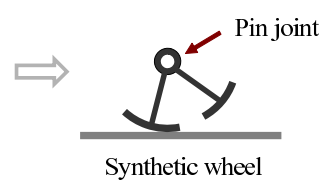

Fig. 1. Wheel, rimless wheel, and synthetic wheel. 


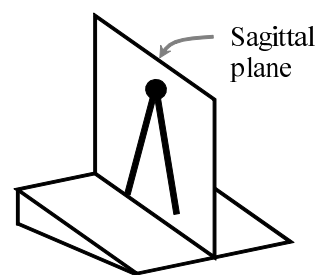

(a) Walking model

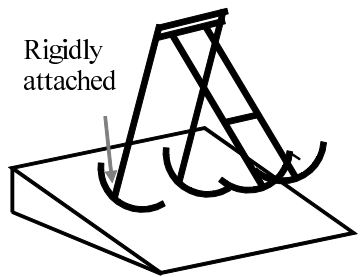

(b) Physical walker

Fig. 2. 2D Passive Biped Walker.
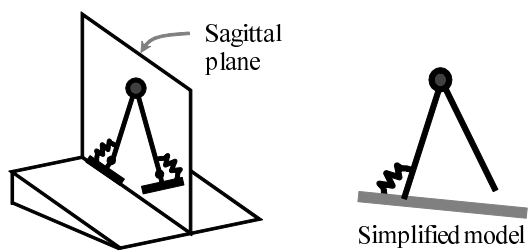

Fig. 3. A 2D straight-legged passive walker with flat feet and ankle springs (Wisse et al., 2006).

\subsection{Rimless Wheel}

A rimless wheel can be obtained by simply removing the rim from the wheel as shown in Fig. 1. One of the features captured by the rimless wheel is the stance leg motion which acts as an inverted pendulum motion. The other feature is the heel strike when the swing leg touches the ground. The rimless wheel has a periodic motion for a given slope angle(McGeer, 1990) whose stable region is very large(Wisse et al., 2005). If the initial rolling speed is sufficiently large and the slope angle is large enough corresponding to the relative angle between the spokes, the rimless wheel never falls forward, and converges to the equilibrium motion(McGeer, 1990; Wisse et al., 2005). This remarkable feature is used to strengthen the stability of passive walkers (Ikemata et al., 2006).

\subsection{Synthetic Wheel}

Passive motion of the swing leg can be explained by the synthetic wheel model(McGeer, 1990) as shown in Fig. 1. In this model, the rim was not removed. The rim was cut between the spokes, and all but two of the spokes removed. A pin joint and a large point mass were put at the hub, i.e. the hip. If the leg mass is assumed to be negligible compared to the hip mass, the swing leg motion will not disturb the stance leg motion. The stance leg rolls at a constant speed on the level floor because it is part of a wheel. McGeer showed that initial conditions exist, such that the synthetic wheel exhibits periodic motion(McGeer, 1990). The step period of the synthetic wheel is determined solely by the free pendulum period of the swing leg.

\subsection{D Passive Biped Walker}

Following the study of rimless wheel and the synthetic wheel model, McGeer increased the complexity of the biped model. A kneeless passive biped walker is similar to a synthetic wheel model, however allows for variation of parameters, e.g., radius of the arc feet and location of the leg mass(McGeer, 1990). McGeer also built several physical passive walkers, with and without knees(McGeer, 1990; 1993). The motion of the McGeer's passive walkers is 


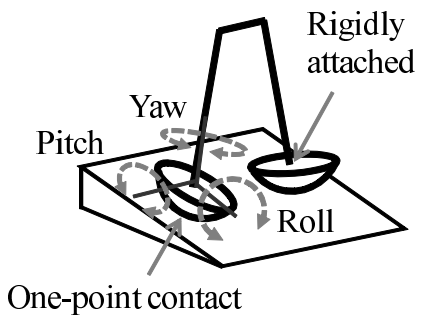

Fig. 4. A 3D passive biped walker

constrained against falling over sideways, as in numerical simulation models. The motion is only in the fore and aft, and vertical, i.e., sagittal, planes as shown in Fig. 2 (a). McGeer and his imitators used four legs, with each set of two legs connected so that they moved identically, to constrain the motion of the walker to the sagittal plane(McGeer, 1990) as indicated in Fig. 2 (b). The physical two-dimensional walkers without knees have a problem of foot scuffing at midstance. Stepping stones make clearance for the swing foot. Shortening the swing leg by lifting the feet via a lead screw mechanism with small motors is an alternative solution(McGeer, 1990). Two-dimensional passive walkers exhibit walking stability on a shallow slope.

Goswami et al. studied the compass model (Goswami et al., 1996), which consists of two straight legs connected by a frictionless hinge at the hip, devoid of actuators and control. The mass is at the hip and legs and the motion is constrained to the sagittal plane. Goswami et al. also showed that the compass model can exhibit period-doubling bifurcation, eventually leading to apparently chaotic gaits, by increasing the slope angle(Goswami et al., 1996; 1998). Garcia et al. introduced the simplest walking model (Garcia et al., 1998), which is similar to the compass model, except that the leg mass is located at the tip of the leg, and the hip mass is much larger than the foot mass. The assumption of negligible leg mass makes the motion of a swinging leg not affect the motion of the hip. The simplest passive walking model still walks stably on a shallow slope (Garcia et al., 1998), although the basin of attraction of the simplest walking model is very small (Schwab and Wisse, 2001). The simplest model exhibits period doubling bifurcations, leading to apparently chaotic gaits, as with the compass model (Garcia et al., 1998). Wisse et al. studied a 2D straight-legged passive walker with flat feet and ankle springs by simplifying the interaction of the spring and the foot (Wisse et al., 2006) as indicated in Fig. 3. The stance leg with the foot and ankle springs is modeled as a point foot with a torsional spring between the stance leg and the floor. Wisse et al. showed that arc-shaped feet rigidly connected to the legs and flat feet with ankle springs have a similar effect on the disturbance behavior in the simple 2D passive walking model (Wisse et al., 2006). Since McGeer's work, a few passive walkers that exhibit stable walking have been constructed. Garcia et al. copied McGeer's 2D kneed passive walker and performed detailed analysis of the gaits(Garcia, 1999; Garcia et al., 2000). Ikemata et al. used a stopper to maintain a constant inter-leg angle at heel strike(Ikemata et al., 2006). The stopper enabled the 2D kneed passive walker obtain high stability. The passive walker can walk on a treadmill for 35 minutes with 4010 steps. 


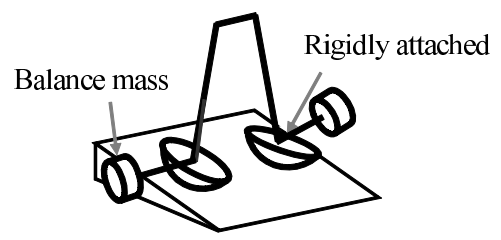

Fig. 5. A simple 3D passive biped walker with laterally extended balance bars (Coleman and Ruina, 1998).

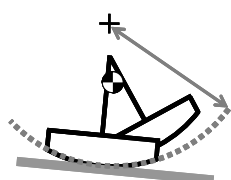

(a) Pitch motion (sagittal plane)

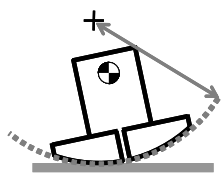

(b) Roll motion (frontal plane)

Fig. 6. A simple 3D passive biped walker with arc-shaped feet whose center of the radius of curvature is higher than the center of the total mass (Tedrake et al., 2004; Tedrake, 2004).

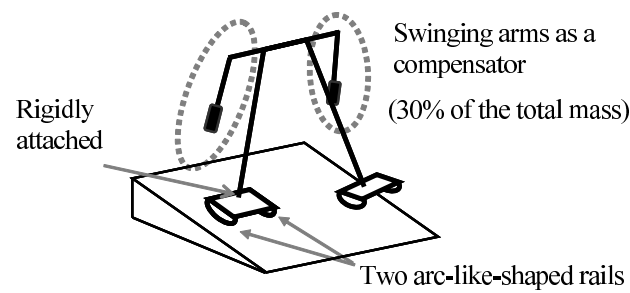

Fig. 7. The most sophisticated three-dimensional passive walker(Collins et al., 2001). It has several non human-like features, e.g., stiff ankles, heavy arms, and out and forward arm motion.

\subsection{Scope of This Study}

We have developed simple 3D passive walkers that can take longer steps and walk faster than other simple 3D passive walkers with arc-shaped feet (Narukawa et al., 2008; 2009a;b). The main feature of our 3D passive walkers is its foot and ankle design; it has flat feet with ankle springs instead of arc-shaped feet rigidly attached to the legs. Experimental results have shown that flat feet with ankle springs stabilize the yaw motion, and our 3D passive biped walker can take longer steps and walk faster than simple arc-footed 3D biped walkers because of its flat feet and ankle springs (Narukawa et al., 2008; 2009a).

This study investigates the effects of torsional spring stiffness on the pitch motion at the ankle joints of the developed walker. Experimental tests are performed to prove that torsional spring stiffness affects the overall motion of the walker and selecting springs with appropriate torsional spring stiffness aids in reducing the oscillating motion of the feet induced by the impact with the ground. 


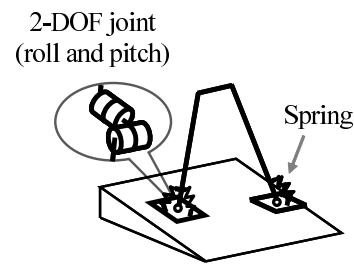

Fig. 8. Our simple 3D passive biped walker with flat feet and ankle springs (Narukawa et al., 2008; 2009a).

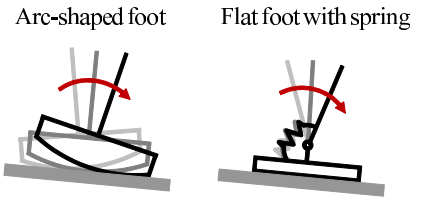

(a) Pitch motion

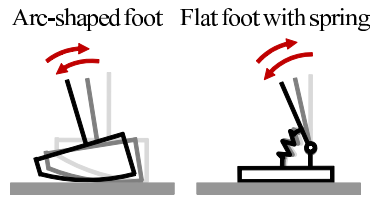

(b) Roll motion

Fig. 9. Motion of a passive walker with flat feet and ankle springs compared to a passive walker with arc-shaped feet rigidly attached to the legs. For clarity, only the stance leg is shown.

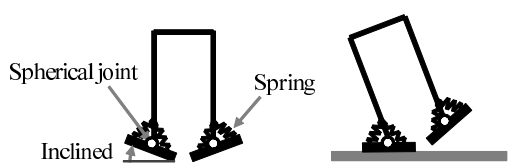

Fig. 10. A 3D passive biped walker with sprung ankles and flat feet. The initial spring tension induces an inclined roll angle (Kinugasa et al., 2008).

\section{3D Passive Biped Walker}

Passive walking has been studied mainly in two dimensions, i.e., the motion is constrained to the sagittal plane e.g. (Garcia et al., 2000; 1998; Goswami et al., 1996; Ikemata et al., 2006; McGeer, 1990; Schwab and Wisse, 2001). Almost all of the physical passive walkers have arc-shaped feet rigidly connected to the legs because the arc-shaped feet can handle the disturbance behavior of the walkers better than point feet (Wisse and van Frankenhuyzen, 2003). Although 2D passive walkers demonstrate that passive leg dynamics can provide stable walking, stable 3D passive walking remains a challenge because of unstable roll and yaw motions as shown in Fig. 4. Roll is rotation about an axis in the direction of motion, and pitch is that about an axis perpendicular to that direction.

\subsection{Simulation Studies}

After studying 2D passive walkers, McGeer demonstrated 3D passive walking without constraining the motion to the sagittal plane (McGeer, 1993). This walker has arc-shaped feet, similar to those of 2D passive walkers. However McGeer only found unstable motion in 3D passive walking and a physical 3D passive walker was not reported. Numerical simulation of three-dimensional passive walking is usually found only with unstable motions (Coleman 


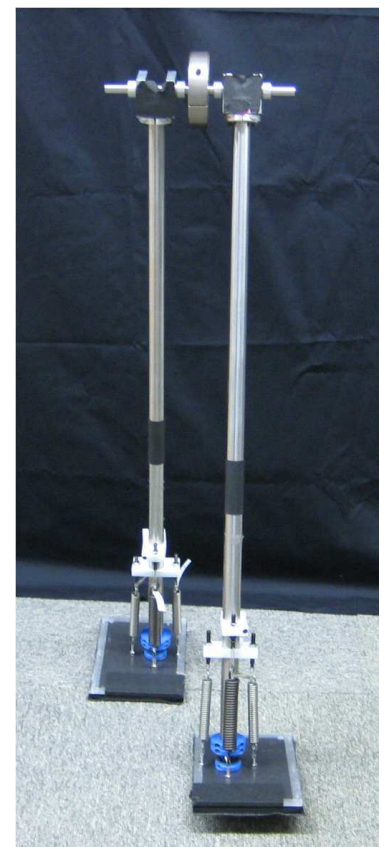

(a) Front view

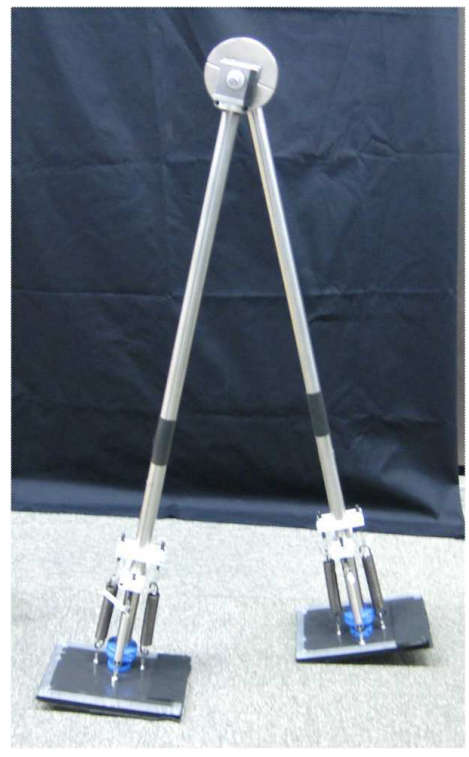

(b) Side view

Fig. 11. A simple 3D straight-legged passive biped walker with flat feet and ankle springs.

and Ruina, 1998; McGeer, 1993). Adolfsson et al. studied three-dimensional passive walking by changing the mechanism configuration from a planar passive walking model to a threedimensional passive walking model (Adolfsson et al., 2001). Gait stability was investigated under parameter variations. Stable gaits of the three-dimensional model were found only when the feet were large enough to overlap. Wisse et al. proposed a 3D biped with a pelvic body as a passive-dynamic compensator for unstable yawing and rolling motion (Wisse et al., 2001). Wisse et al. studied a 3D passive walker having cylinder-shaped feet with ankle joints that kinematically couple roll to yaw (Wisse and Schwab, 2005).

\subsection{Physical Passive Walkers}

Coleman and Ruina built a simple two-leg passive walker with rounded feet attached rigidly to the legs, and laterally extended balance bars as shown in Fig. 5 (Coleman and Ruina, 1998). Although it cannot stand still, it can walk stably in three dimensions. However it takes only very short steps, resulting in low walking speed. Although Coleman et al. found stable motion of a 3D passive biped using an optimization method to find a set of walker parameters that produced stable walking (Coleman et al., 2001); however, the parameters are far from those of a physical prototype that exhibits stable motion. One of the simplest 3D passive biped walkers was built by Tedrake et al.(Tedrake et al., 2004; Tedrake, 2004). It has large arc-shaped feet, whose center of the radius of curvature is higher than the center of the total mass, which allows it to stand (Fig. 6). The contact with the ground occurs only at a point, and provides insufficient friction against unstable yaw motion, thus it can take 


\begin{tabular}{ccc|ccc}
\hline Parameter & Unit & Value & Parameter & Unit & Value \\
\hline$m_{L}$ & $\mathrm{~kg}$ & 0.70 & $l_{F F}$ & $\mathrm{~m}$ & 0.12 \\
$m_{H}$ & $\mathrm{~kg}$ & 0.88 & $l_{F L}$ & $\mathrm{~m}$ & 0.18 \\
$l_{L}$ & $\mathrm{~m}$ & 0.74 & $l_{F T}$ & $\mathrm{~m}$ & 0.09 \\
$l_{A}$ & $\mathrm{~m}$ & 0.03 & $r_{L}$ & $\mathrm{~m}$ & 0.49 \\
$l_{H}$ & $\mathrm{~m}$ & 0.09 & $I_{x x}$ & $\mathrm{~kg} \cdot \mathrm{m}^{2}$ & 0.07 \\
$l_{F W}$ & $\mathrm{~m}$ & 0.08 & $I_{y y}$ & $\mathrm{~kg} \cdot \mathrm{m}^{2}$ & 0.07 \\
\hline
\end{tabular}

Table 1. Parameters of the physical 3D passive walker

very small strides only. The most sophisticated three-dimensional passive walker was built by Collins et al.(Collins et al., 2001) as shown in Fig. 7. They did not use simulation studies because numerical simulations of three-dimensional passive walkers are difficult, mainly due to collisions between the swing foot and the ground, and the frictional phenomena between the stance foot and the ground during gaits. Their 3D passive walker was improved by trial and error during experimental study. To reduce the unstable yaw motion, swing arms were attached to the counter side legs. Although the walker has several non human-like features, e.g., stiff ankles, heavy arms (accounting for $30 \%$ of the total mass of the walker), and out and forward arm motion, the three-dimensional passive biped walks stably at about $0.5 \mathrm{~m} / \mathrm{s}$ and exhibits impressively human-like motion.

\section{3D Passive Biped Walker with Flat Feet and Ankle Springs}

We investigated simple 3D straight-legged passive walking with flat feet and ankle springs, as shown in Fig. 8, to overcome the limitations of arc-footed walkers, while maintaining mechanical simplicity (Narukawa et al., 2008; 2009a). The walker is composed of a hip, two straight legs, and two feet. The walker does not have knees and the yaw degree of freedom at the ankles so as to maintain a small number of degrees of freedom. The ankles have two degrees of freedom in roll and pitch motion. The proposed 3D passive walker do not have a compensator, such as swinging arms (Collins et al., 2001) or a pelvic body (Wisse et al., 2001), for unstable motion.

The main feature of the 3D passive walker is the flat feet with ankle springs, which enable the walker to mimic the motion of a 3D straight-legged passive walker with rigidly attached arc-shaped feet (Tedrake et al., 2004), as shown in Fig. 9, while providing sufficient friction torque against yaw. The flat feet and ankle springs allow the 3D passive walker to take long steps and walk faster than passive walkers with arc-shaped feet by stabilizing the unstable yaw motion (Narukawa et al., 2008; 2009a). Kinugasa et al. built a 3D straight-legged passive walker with flat feet and springs attached to ankles; it is $0.6 \mathrm{~m}$ tall (Kinugasa et al., 2008). The initial spring tension induces an inclined roll angle, as indicated in Fig. 10. Their 3D biped walks stably, but takes only very short steps of about $0.04 \mathrm{~m}$.

\section{Physical 3D Passive Biped Walker with Flat Feet and Ankle Springs}

A physical 3D passive biped walker was constructed, as shown in Fig. 11, to investigate the proposed method(Narukawa et al., 2008; 2009a). 


\begin{tabular}{cccc}
\hline Parameter & Unit & \multicolumn{2}{c}{ Value } \\
& & roll & pitch \\
\hline$a$ & $\mathrm{~m}$ & 0.025 & 0.025 \\
$b$ & $\mathrm{~m}$ & 0.030 & 0.030 \\
$h$ & $\mathrm{~m}$ & 0.112 & 0.142 \\
$s$ & $\mathrm{~m}$ & 0.103 & 0.130 \\
$k$ & $\mathrm{~N} / \mathrm{m}$ & 4900 & see Fig. 14 \\
\hline
\end{tabular}

Table 2. Parameters of the foot and ankle design of the 3D passive walker

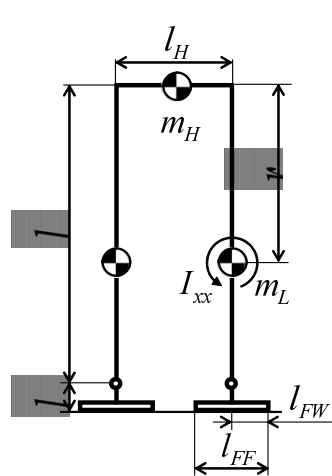

(b) Front view

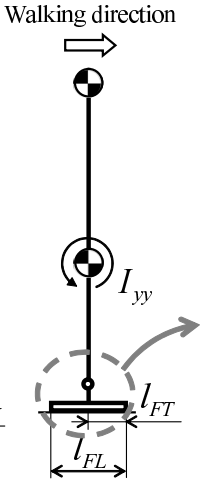

(a) Side view

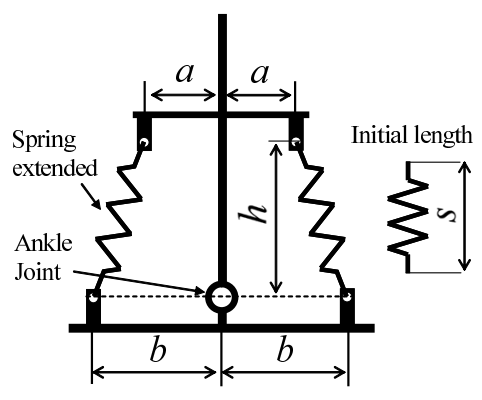

(c) Foot and ankle springs

Fig. 12. Notations for physical parameters of the $3 \mathrm{D}$ passive walker with flat feet and ankle springs.

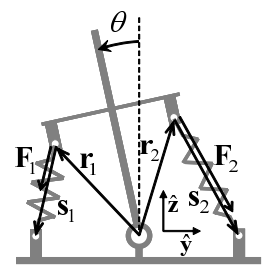

Fig. 13. A model of the foot and ankle springs. The tension springs are attached to the leg and the foot. The torque about the joint is produced by the springs.

\subsection{Walker Parameters}

Table 1 shows the parameter values of the physical walker. Figure 12 indicates the notations. The legs are symmetric, and the hip is assumed to be a point mass. The length of the legs, width of the hip, and mass distribution were determined as follows. First, we decided to make the legs about $0.8 \mathrm{~m}$ long, based on the length of human legs, other human-like passive walkers (Collins et al., 2001), and passive-based walkers (Collins and Ruina, 2005; Collins et al., 2005). The period of the swing leg motion is about $1.7 \mathrm{~s}$. The width of the hip is as short as possible. Extra mass is added to the hip to increase the height of the center of mass 


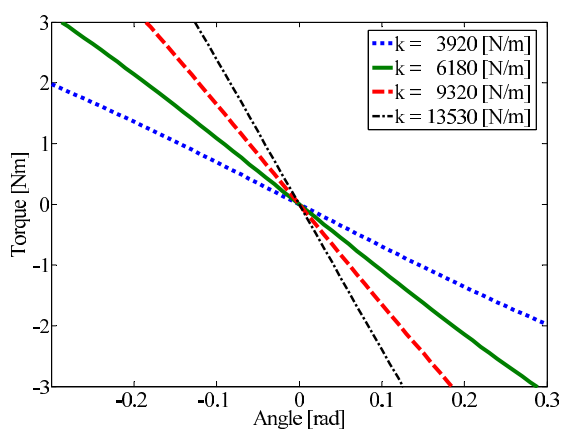

Fig. 14. Relationship between the pitch angle and the torque applied by the springs.

of the walker. The 3D passive walker weighs $2.3 \mathrm{~kg}$, and its center of mass measured from the ground is about $0.5 \mathrm{~m}$.

\subsection{Torsional Spring Constant at the Ankle Joint}

The flat foot and the leg are joined by a universal joint that has two degrees of freedom. Sponge sheets are attached to the soles of the feet to increase friction. The torsional spring effect is realized by using a pair of tension springs. Tension springs are attached to the feet and the legs to produce torque about the joint. The effect of the tension springs is simply calculated as follows(Narukawa et al., 2008; 2009a). First we assume that the roll and pitch motion are each isolated. The pitch motion is described as shown in Fig. 13. The forces produced by the springs are

$$
\begin{gathered}
\left|\mathbf{F}_{1}\right|=k\left(\left|\mathbf{s}_{1}\right|-s\right), \\
\left|\mathbf{F}_{2}\right|=k\left(\left|\mathbf{s}_{2}\right|-s\right)
\end{gathered}
$$

where $k$ is the spring constant and $s$ is the initial length of the spring. We assume that the spring force obeys Hooke's law. The torque produced by the spring forces becomes

$$
\mathbf{T}=\sum_{i=1}^{2} \mathbf{r}_{i} \times \mathbf{F}_{i}=\sum_{i=1}^{2} k\left(\left|\mathbf{s}_{i}\right|-s\right) \mathbf{r}_{i} \times \frac{\mathbf{s}_{i}}{\left|\mathbf{s}_{i}\right|} .
$$

Figure 13 provides the necessary notation. Table 2 shows the values of the parameters of the physical walker. Then

$$
\begin{gathered}
\mathbf{r}_{1}=(-h \sin \theta-a \cos \theta) \hat{\mathbf{y}}+(h \cos \theta-a \sin \theta) \hat{\mathbf{z}}, \\
\mathbf{r}_{2}=(-h \sin \theta+a \cos \theta) \hat{\mathbf{y}}+(h \cos \theta+a \sin \theta) \hat{\mathbf{z}}, \\
\mathbf{s}_{1}=-\mathbf{r}_{1}-b \hat{\mathbf{y}}, \\
\mathbf{s}_{2}=-\mathbf{r}_{2}+b \hat{\mathbf{y}} .
\end{gathered}
$$

$\hat{\mathbf{y}}$ and $\hat{\mathbf{z}}$ are unit vectors illustrated by Fig. 13 .

The torsional spring constant for the roll motion at the stance ankle is obviously an important factor in enabling the straight-legged walker to rock adequately from side to side to avoid problematic scuffing of the swing leg and allowing it to swing forward. The study of the roll and pitch motions with ankle springs clarifies the effect of the springs so that the roll and pitch 


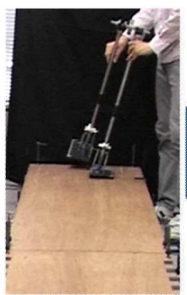

(a) Launch

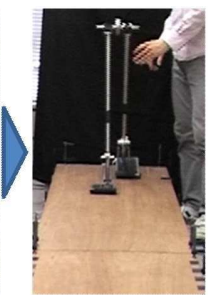

(b) 0th step

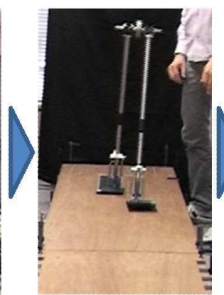

(c) 1st step

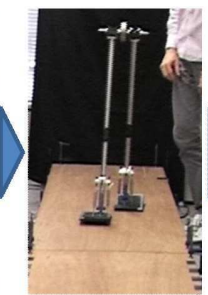

(d) 2nd step

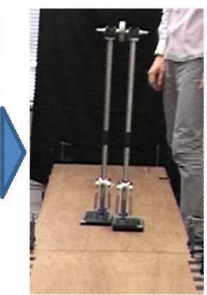

(e) Not counted

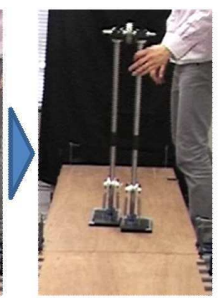

(f) End the trial

Fig. 15. Counting steps in passive walking. The first landing is counted as the zeroth step and the second landing as the first.

\begin{tabular}{cccccc}
\hline Successful Steps & \multicolumn{5}{c}{ Number of times (in 100 launch) } \\
& $3920 \mathrm{~N} / \mathrm{m}$ & $6180 \mathrm{~N} / \mathrm{m}$ & $9320 \mathrm{~N} / \mathrm{m}$ & $13530 \mathrm{~N} / \mathrm{m}$ & $\infty \mathrm{N} / \mathrm{m}$ \\
\hline 0 & 40 & 12 & 7 & 12 & 40 \\
1 & 51 & 68 & 68 & 70 & 49 \\
2 & 9 & 14 & 19 & 12 & 10 \\
3 & 0 & 3 & 6 & 5 & 1 \\
4 & 0 & 3 & 0 & 1 & 0 \\
\hline Expectation & 0.78 & 1.17 & 1.24 & 1.13 & 0.72 \\
\hline
\end{tabular}

Table 3. Experimental result for each pitch angle spring constant

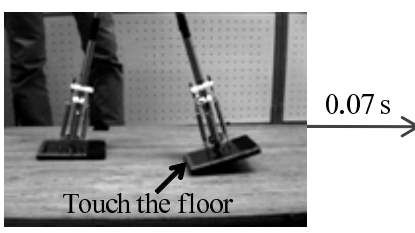

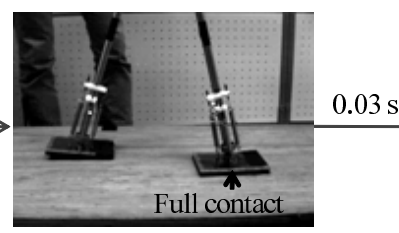

(a) $3920 \mathrm{~N} / \mathrm{m}$
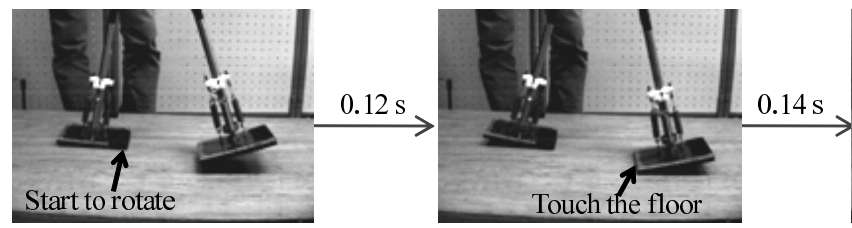

(b) $9320 \mathrm{~N} / \mathrm{m}$

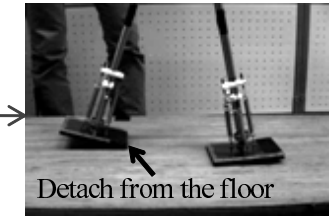

Detach from the floor

Fig. 16. Comparison of the foot motion for varying spring stiffness

motions are coordinated. From (Narukawa et al., 2009a), the value of the spring constant of the extension spring for roll motion is determined to be $4900 \mathrm{~N} / \mathrm{m}$ with a torsional spring stiffness of about $9 \mathrm{~N}-\mathrm{m} / \mathrm{rad}$. Figure 14 shows the relationship between the ankle angle and the torque applied by the springs for pitch motion when the spring constant is 3920,6180 , 9320 , and $13530 \mathrm{~N} / \mathrm{m}$. 


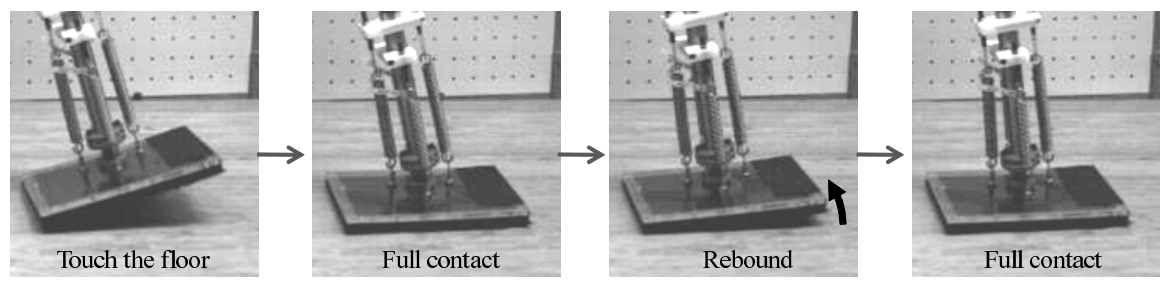

Fig. 17. Rebound at foot impact $(3920 \mathrm{~N} / \mathrm{m})$

\section{Experimental Results}

Experimental conditions were as follows. The slope angle was about 1.6 degrees; the slope was about $3.6 \mathrm{~m}$ long and $0.6 \mathrm{~m}$ wide. In each trial, the walker was started by hand from the top of the slope.

\subsection{Successful Steps at Different Spring Constants for Pitch Motion}

Figure 15 shows how we count the number of realized steps. After the launch in Fig. 15 (a), first landing is counted as the zeroth step and second landing is the first step, as shown in Fig. 15 (b) and (c). When the swing leg lands and the heel is behind the toe of the stance foot, we do not count the landing as a successful step and regard the walking as a failure, as shown in Fig. 15 (e) and (f). In some situations, we do not count a landing as a successful step even though the swing leg lands in front of the stance leg. Table 3 shows the experimental results. We changed pitch spring constant of the ankles and launched the biped walker 100 times for each settings. The spring constant $\infty[\mathrm{N} / \mathrm{m}]$ means that we locked the ankle pitch movements with wires instead of springs. However, the feet are not ideal rigid bodies because we attached sponge sheets to the soles of the feet. The result shows that a medium spring constant effectively stabilizes passive walking. High and low spring constants are ineffective because unsuccessful trials ( 0 step) occurred frequently, producing a low expectation of successful steps.

\subsection{Foot Motion with the Ground}

The spring stiffness of the pitch motion affects the foot motion with the floor. Figure 16 compares the foot motion for different spring stiffness. When the spring constant is $3920 \mathrm{~N} / \mathrm{m}$, which is equal to a torsional spring stiffness of about $7 \mathrm{Nm} / \mathrm{rad}$, the foot of the stance leg remains in full contact with the floor until the heel of the swing leg touches the floor. Next, the front foot fully impacts the floor and rebound occurs, as indicated in Fig. 17. On the other hand, when the torsional spring stiffness is large, e.g., the spring constant is $9320 \mathrm{~N} / \mathrm{m}$, the rotation of the stance foot around the toe occurs before the swing foot touches the floor and the rebound after the full contact of the front foot is dramatically reduced. A high torsional spring stiffness for pitch motion leads to a smooth transition at the exchange of the stance leg. When the torsional spring stiffness is very large, the pitch angle is always 0 , the stance foot almost always rotates around the heel or toe, and rebound does not occur.

\section{Conclusions}

This paper presents a simple 3D passive biped walker with flat feet and ankle springs. Experimental tests were performed to investigate the effects of torsional spring stiffness on the pitch 
motion at the ankle joints of the walker. When the spring stiffness is low, oscillating motion is induced by the impact of the feet with the ground. Experimental results showed that using springs with appropriate torsional spring stiffness effectively reduces the oscillating motion. The rebound of the front foot after full contact with the ground reduces dramatically with appropriate torsional spring stiffness. Appropriate stiffness enables the biped walker to walk smoothly and also stabilizes the walker. However, when the spring stiffness is either high or low, it become difficult for the walker to walk.

\section{ACKNOWLEDGMENTS}

This work was supported in part by Grant in Aid for the Global Center of Excellence Program for "Center for Education and Research of Symbiotic, Safe and Secure System Design" from the Ministry of Education, Culture, Sports, Science and Technology in Japan.

\section{References}

Adolfsson, J., Dankowicz, H., and Nordmark, A. (2001). 3D passive walkers: Finding periodic gaits in the presence of discontinuities. Nonlinear Dynamics, 24(2):205-229.

Coleman, M. J., Garcia, M., Mombaur, K., and Ruina, A. (2001). Prediction of stable walking for a toy that cannot stand. Physical Review E, 64(2):22901-.

Coleman, M. J. and Ruina, A. (1998). An uncontrolled walking toy that cannot stand still. Physical Review Letters, 80(16):3658-3661.

Collins, S. and Ruina, A. (2005). A bipedal walking robot with efficient and human-like gait. Proceedings of the 2005 IEEE International Conference on Robotics and Automation, pages 1983-1988.

Collins, S., Ruina, A., Tedrake, R., and Wisse, M. (2005). Efficient bipedal robots based on passive-dynamic walkers. Science, 307(5712):1082-1085.

Collins, S., Wisse, M., and Ruina, A. (2001). A three-dimensional passive-dynamic walking robot with two legs and knees. International Journal of Robotics Research, 20(7):607615.

Garcia, M. (1999). Stability, scaling, and chaos in passive-dynamic gait models. PhD thesis, Cornell University.

Garcia, M., Chatterjee, A., and Ruina, A. (2000). Efficiency, speed, and scaling of twodimensional passive-dynamic walking. Dynamics and Stability of Systems, 15(2):75-99.

Garcia, M., Chatterjee, A., Ruina, A., and Coleman, M. (1998). The simplest walking model: Stability, complexity, and scaling. Journal of Biomechanical Engineering-Transactions of the ASME, 120(2):281-288.

Goswami, A., Espiau, B., and Keramane, A. (1996). Limit cycles and their stability in a passive bipedal gait. Proceedings of the 1996 IEEE International Conference on Robotics and Automation, pages 246-251.

Goswami, A., Thuilot, B., and Espiau, B. (1998). A study of the passive gait of a compasslike biped robot: Symmetry and chaos. International Journal of Robotics Research, 17(12):1282-1301.

Hobbelen, D. G. E. and Wisse, M. (2007). Limit cycle walking. In Hackel, M., editor, Humanoid Robots: Human-like Machines, pages 277-294. I-Tech Education and Publishing, Vienna, Austria. 
Ikemata, Y., Sano, A., and Fujimoto, H. (2006). A physical principle of gait generation and its stabilization derived from mechanism of fixed point. Proceedings of the 2006 Conference on International Robotics and Automation, pages 836-841.

Kinugasa, T., Kotake, K., Haruki, T., Tanaka, H., and Yoshida, K. (2008). 3D passive dynamic walker with sprung ankle and flat foot -a design method by natural frequency index without yaw and roll compensator-. Proceedings of the 2008 JSME Conference on Robotics and Mechatronics, pages 1P1-B12-.

Kuo, A. D. (1999). Stabilization of lateral motion in passive dynamic walking. International Journal of Robotics Research, 18(9):917-930.

McGeer, T. (1990). Passive dynamic walking. International Journal of Robotics Research, 9(2):6282.

McGeer, T. (1993). Passive dynamic biped catalogue, 1991. In Chatila, R. and Hirzinger, G., editors, Experimental Robotics II: The 2nd International Symposium, Toulouse, France, June 25-27 1991, pages 465-490. Springer-Verlag.

McMahon, T. A. (1984). Mechanics of locomotion. International Journal of Robotics Research, 3(2):4-28.

Narukawa, T., Yokoyama, K., Takahashi, M., and Yoshida, K. (2008). A simple 3D straightlegged passive walker with flat feet and ankle springs. IEEE/RSJ International Conference on Intelligent Robots and Systems, pages 2952-2957.

Narukawa, T., Yokoyama, K., Takahashi, M., and Yoshida, K. (2009a). Design and construction of a simple 3D straight-legged passive walker with flat feet and ankle springs. JSME Journal of System Design and Dynamics, 3(1):1-12.

Narukawa, T., Takahashi, M., and Yoshida, K. (2009b). Design and Stability Analysis of a 3D Rimless Wheel with Flat Feet and Ankle Springs. JSME Journal of System Design and Dynamics, 3(3):258-269.

Schwab, A. L. and Wisse, M. (2001). Basin of attraction of the simplest walking model. Proceedings of the ASME Design Engineering Technical Conference, pages DETC2001/VIB21363-.

Tedrake, R., Zhang, T. W., Fong, M.-F., and Seung, H. S. (2004). Actuating a simple 3D passive dynamic walker. Proceedings of the IEEE International Conference on Robotics and Automation, 2004(5):4656-4661.

Tedrake, R. L. (2004). Applied Optimal Control for Dynamically Stable Legged Locomotion. PhD thesis, Massachusetts institute of technology.

Wisse, M., Hobbelen, D. G. E., Rotteveel, R. J. J., Anderson, S. O., and Zeglin, G. J. (2006). Ankle springs instead of arc-shaped feet for passive dynamic walkers. Proceedings of the IEEE-RAS International Conference on Humanoid Robots, pages 110-116.

Wisse, M. and Schwab, A. L. (2005). Skateboards, bicycles, and three-dimensional biped walking machines: Velocity-dependent stability by means of lean-to-yaw coupling. International Journal of Robotics Research, 24(6):417-429.

Wisse, M., Schwab, A. L., van der Linde, R. Q., and van der Helm, F. C. T. (2005). How to keep from falling forward: Elementary swing leg action for passive dynamic walkers. IEEE Transactions on Robotics, 21(3):393-401.

Wisse, M., Schwab, A. L., and vander Linde, R. Q. (2001). A 3D passive dynamic biped with yaw and roll compensation. Robotica, 19:275-284.

Wisse, M. and van Frankenhuyzen, J. (2003). Design and construction of mike; a 2D autonomous biped based on passive dynamic walking. Proceedings of the Second International Symposium on Adaptive Motion of Animals and Machines, pages 4-8. 


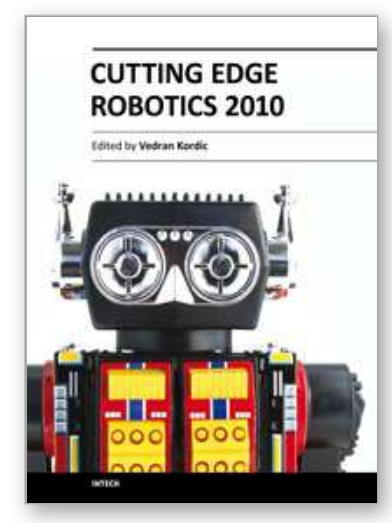

\author{
Cutting Edge Robotics 2010 \\ Edited by Vedran Kordic
}

ISBN 978-953-307-062-9

Hard cover, 440 pages

Publisher InTech

Published online 01, September, 2010

Published in print edition September, 2010

Robotics research, especially mobile robotics is a young field. Its roots include many engineering and scientific disciplines from mechanical, electrical and electronics engineering to computer, cognitive and social sciences. Each of this parent fields is exciting in its own way and has its share in different books. This book is a result of inspirations and contributions from many researchers worldwide. It presents a collection of a wide range of research results in robotics scientific community. We hope you will enjoy reading the book as much as we have enjoyed bringing it together for you.

\title{
How to reference
}

In order to correctly reference this scholarly work, feel free to copy and paste the following:

Terumasa Narukawa, Kazuto Yokoyama, Masaki Takahashi and Kazuo Yoshida (2010). An Experimental Study of Three-Dimensional Passive Dynamic Walking with Flat Feet and Ankle Springs, Cutting Edge Robotics 2010, Vedran Kordic (Ed.), ISBN: 978-953-307-062-9, InTech, Available from:

http://www.intechopen.com/books/cutting-edge-robotics-2010/an-experimental-study-of-three-dimensionalpassive-dynamic-walking-with-flat-feet-and-ankle-springs

\section{INTECH}

open science | open minds

\section{InTech Europe}

University Campus STeP Ri Slavka Krautzeka 83/A 51000 Rijeka, Croatia Phone: +385 (51) 770447

Fax: +385 (51) 686166 www.intechopen.com

\section{InTech China}

Unit 405, Office Block, Hotel Equatorial Shanghai No.65, Yan An Road (West), Shanghai, 200040, China 中国上海市延安西路65号上海国际贵都大饭店办公楼405单元 Phone: +86-21-62489820

Fax: +86-21-62489821 
(C) 2010 The Author(s). Licensee IntechOpen. This chapter is distributed under the terms of the Creative Commons Attribution-NonCommercialShareAlike-3.0 License, which permits use, distribution and reproduction for non-commercial purposes, provided the original is properly cited and derivative works building on this content are distributed under the same license. 